\title{
Bilateral obturator herniae and associated femoral hernia
}

\author{
R. M. WATKins \\ F.R.C.S.
}

\author{
R. D. LEACH \\ F.R.C.S.
}

\author{
H. ELLIS \\ D.M., M.Ch., F.R.C.S. \\ Surgical Unit, Westminster Medical School, London SWIP 2AP
}

\begin{abstract}
Summary
A case of a strangulated obturator hernia associated with a strangulated femoral hernia is reported. The development of obturator herniae and their association with other herniae are discussed.
\end{abstract}

\section{Introduction}

Obturator hernia is a rare cause of intestinal obobstruction usually seen in elderly emaciated women. An apparently unique case of obturator herniae and a femoral hernia is reported.

\section{Case}

A well nourished 43-year-old female presented with intestinal obstruction and a tender irreducible left femoral hernia. A viable loop of ileum was released from the hernia via a low inguinal approach and the small bowel proximal and distal were carefully examined. The femoral hernia was repaired and the patient made a slow but steady recovery, being discharged on the sixth postoperative day.

Eight days after her discharge, symptoms of further intestinal obstruction recurred and the patient was admitted for observation. No mass was palpable in the left iliac fossa or groin but bowel sounds were obstructive and fluid levels with dilated small bowel loops were present on erect abdominal $\mathrm{X}$-rays. The obstruction did not settle on conservative treatment and a laparotomy was performed. This revealed a loop of ileum strangulated within a large left obturator hernia medial to the vessels and nerve. A small bowel resection was performed and the defect repaired with peritoneum. On the right side an empty obturator hernia was present, medial to the vessels and nerve, which admitted the terminal phalanx of an index finger. This sac was reduced and excised and the defect closed with interrupted nylon sutures. Postoperatively the patient made an uneventful recovery.

\section{Comment}

Over 500 cases of obturated hernia have b\&n reported since de Ronsil first recognized the cofdition in 1722. They are 6 times more commonopn females than males and are usually seen in seventh or eighth decade. A history of multiple pregnancies and obesity followed by dramatic weight loss is often present. Most patients present with intestinal obstruction or vague abdominal pains, and about $50 \%$ have pain on the m\&f+al side of the knee (Howship-Romberg sign) dir to pressure on the obturator nerve. Few obtum herniae are diagnosed before surgery.

Gray and Skandalakis (1978) describe 3 stagesain the development of an obturator hernia: (1) फ़िe entrance of pre-peritoneal fat; (2) the entrance - of peritoneum, and (3) the entrance of a viscus info the obturator canal. In the case reported, coexisting second and third stage obturator hernitate were present. Hanley and Hanna (1970) reported 2 cases of bilateral obturator herniae, both in females. Gray records 2 patients with a single obturaţr hernia and a co-existing inguinal or femoral herna. The overall mortality of this rare condition is $13 \%$, most of which is due to cardiorespiratory disease 3 n a group of patients in their seventh or eighth decades.

The present combination of bilateral obturator herniae and a femoral hernia has not been priciviously reported.

\section{References}

Gray, S.W. \& SKandalakis, J.E. (1978) Strangulared obturator hernia. In: Hernia (Ed by L. M. Nyhus $\frac{c}{\mathbb{D}} \&$ H. N. Harkins). J. B. Lippicott, Philadelphia.

HANLEY, J.A. \& HANNA, B.K.B. (1970) Obturator hernija. A report of three cases with strangulation occurring twice in two patients. Journal of the Irish Medical Association, 63, 396. 